Proc. Indian Acad. Sci. (Chem. Sci.), Vol. 105, No. 6, December 1993, pp. 555-562.

(C) Printed in India.

\title{
Sunlight-initiated cycloaddition reactions of the benzene ring
}

\author{
S Y AL-QARADAWI, ${ }^{a}$ D C BLAKEMORE ${ }^{b}$ and A GILBERT*b \\ -Chemistry Department, Qatar University, P O Box 110003, Doha, Qatar \\ ${ }^{b}$ Chemistry Department, The University of Reading, Whiteknights, P O Box 224, Reading, \\ Berkshire, RG6 2AD, UK
}

\begin{abstract}
Both 1,2-(ortho) and 1,3-(meta) cycloaddition of ethenes to the benzene ring can be accomplished using sunlight-initiated chemistry. The process is most effective for arenes which have both electron-donor and electron-acceptor substituents and occurs both inter- and intramolecularly giving an inexpensive and convenient access to complex molecular skeletons from readily available starting materials.
\end{abstract}

Keywords. Sunlight; photocycloaddition; benzocyclobutene; triquinane.

\section{Introduction and brief historical perspective of sunlight-initiated chemistry}

There are many examples, dating back several thousand years, of the importance of sunlight in middle-eastern cultures (Morsi and Abdel-Mottaleb 1991). The study of photochemistry as a science, however, is widely regarded as having its origins during the late 19 th and early 20 th centuries. During this period, numerous reports appeared describing the sunlight-initiated reactions of organic compounds (Singer 1986; Carassiti 1988; Roth 1988; Morsi and Abdel-Mottaleb 1991). For example, Sestini (1866) had published the first paper on the solution photochemistry of santonin and Fritzsche (1867) had observed the photodimerisation of anthracene but referred to the product as a "para body". Liebermann (1877) and Breuer and Zincke (1878) noted the bleaching of the yellow crystals of thymoquinone and 2-phenylnaphtho-1,4quinone respectively in sunlight, and these photoreactions were later shown to be the first observed examples of $(2 \pi+2 \pi)$ cycloadditions of ethenes. By 1902 the cyclobutane nature of the products of the photodimers of ethenes had been established by Riiber (1902), and cis-trans isomerisation of cinnamic acid in sunlight had been reported by Perkin (1881) and by Liebermann (1890). The photoreduction of 1,4-quinones and their light-induced reactions with aldehydes attracted much attention at this time. Such interest in these reactions caused Heinrich Klinger (Klinger and Slandke 1891), one of the principal investigators of these processes, to refer to them as Synthesen durch Sonnenlicht.

It was during this period that Ciamician and Silber at the University of Bologna were carrying out their extensive and thorough investigations into the sunlightinitiated chemistry of organic compounds (Carassiti 1988). Indeed so great was their contribution at that time that Ciamician was later described as "the father of

*For correspondence 
photochemistry" (Turro 1965), and 1900 as the year which "marks the basic date in the history of photochemistry" (Nasini 1929).

Ciamician and Silber were, however, far from being alone in recognising that sunlight could induce such remarkable changes in the molecular structure of organic compounds. One of their more notable fellow pioneers of the time was the Italian chemist Paterno. His major contribution arose from the observation that exposure of solutions of benzaldehyde and 2-methylbut-2-ene to sunlight produced an oxetane (Paterno and Chieffi 1909). The importance of this work was not fully appreciated until much later when Buchi and coworkers greatly extended the scope of the process (Buchi et al 1954) and this reaction is now fittingly known as the "Paterno-Buchi" reaction.

The appointment in 1937 of Alexander Schonberg to the Chair of Chemistry and as Director of the Chemistry Department at the University of Cairo increased interest in the study of sunlight-initiated processes in Egypt. From this time until his return to Berlin in 1958, Schonberg, in collaboration with Mustafa, produced a considerable number of publications describing the outstanding achievements of the Egyptian research group (Singer 1986).

Interest in sunlight-initiated processes during the past 35 years has, however, almost exclusively concentrated on the conversion of solar energy into more versatile energy sources but, at the same time, photochemistry has been shown to be a viable and useful synthetic technique (Horspool 1984; Coyle 1986). As noted recently, however, the photon may be a clean and convenient reagent but it can also be expensive to generate from artificial sources (Hulsdunker et al 1992): hence the growing interest in "solar chemistry" and a desire for Synthesen durch Sonnenlicht.

\section{Results and discussion}

With few exceptions, early studies on sunlight-initiated processes of organic compounds have been concerned with the reactions of ethenes, carbonyl compounds, and diazo and diazonium compounds. In contrast, our interest is in inducing reactions of the benzene ring using sunlight. These studies originate from our investigations in the early 1960 's on the photochemistry of ground-state charge-transfer complexes between dienophiles and benzenoid compounds. The complex between maleic anhydride and benzene absorbs at longer wavelengths than either of the components and "tails" well beyond $290 \mathrm{~nm}$ (Andrews and Keefer 1964). Excitation within this charge-transfer absorption band results in the formation of the 2:1 cycloadduct 1 (Angus and Bryce-Smith 1959). The process can be sensitised by aromatic ketones of triplet energy greater than $275 \mathrm{~kJ} \mathrm{~mol}^{-1}$ (Schenck and Steinmetz 1960) and both sensitised and unsensitised reactions oceur on exposure of the solutions to (Reading)

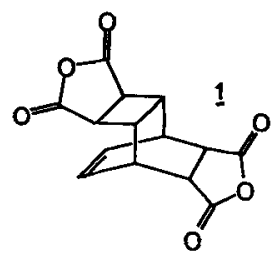




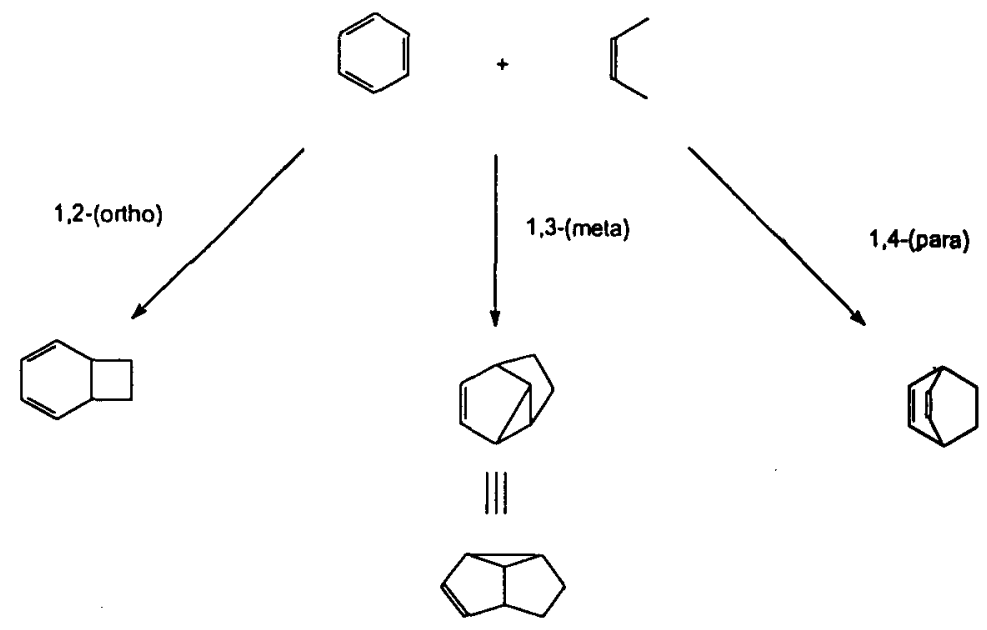

Scheme 1. Modes of photocycloaddition of ethenes to the benzene ring.

sunlight (Bryce-Smith et al 1962). The 2:1 adduct has attracted considerable interest not only because it was the first photoaddition product of the benzene ring reported but also because of its potential to yield condensation polymers of high thermal stability with diamines and because the diimide derivative shows appreciable cytotoxic activity (Lomax and Narayanan 1984).

However, the process is remarkably limited and despite the ready formation of derivatives of 1 from $313 \mathrm{~nm}$ radiation of solutions of maleic anhydride in alkyl benzenes (but not alkoxybenzenes), only the parent compound can be obtained using sunlight initiation: from the other systems maleic anhydride-based polymers are obtained (Bryce-Smith et al 1962). It has now long been recognised that excitation within charge-transfer bands is not a prerequisite for photocycloaddition to the benzene ring, and numerous products derived from 1,2-(ortho), 1,3-(meta), and 1,4-(para) cycloadditions (scheme 1) of ethenes have been reported. The majority of the arenes in these reactions require $254 \mathrm{~nm}$ radiation for excitation and, of course, their reactions are not initiated by sunlight. Recently, we have recognised that a combination of both electron donors and electron acceptors as substitutents on the benzene ring not only provides functionalisation of the carbon skeletons of the cycloadducts, but also, and importantly, leads to a red-shift in the arene absorption beyond $300 \mathrm{~nm}$ while maintaining the lowest $\pi, \pi^{*}$ excited state. These arenes can be directly excited using the shorter wavelength components of sunlight and by employing this simple and inexpensive methodology, functionalised complex carbon skeletons can be readily and conveniently accessed. The following cycloadditions to the benzene ring can all be induced by exposure of solutions of the reactants to sunlight.

\subsection{1,2-(Ortho)-photocycloaddition}

There is a correlation between the preferred mode of cycloaddition (ortho versus meta) of an ethene to the benzene ring and the relative electron donor-electron acceptor characteristics of the addends. This donor-acceptor relationship has been estimated by ionisation potential differences (Bryce-Smith and Gilbert 1977) and more rigorously by the magnitude of the free energy change $\Delta G^{0}$ of electron transfer between 

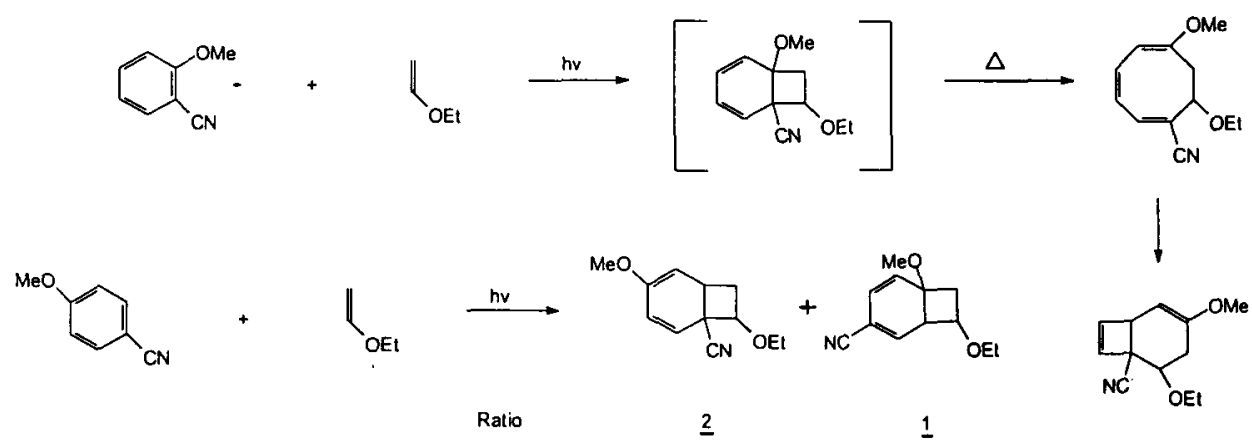

Scheme 2. Cycloaddition of ethyl vinyl ether to 2- and 4-cyanoanisoles.

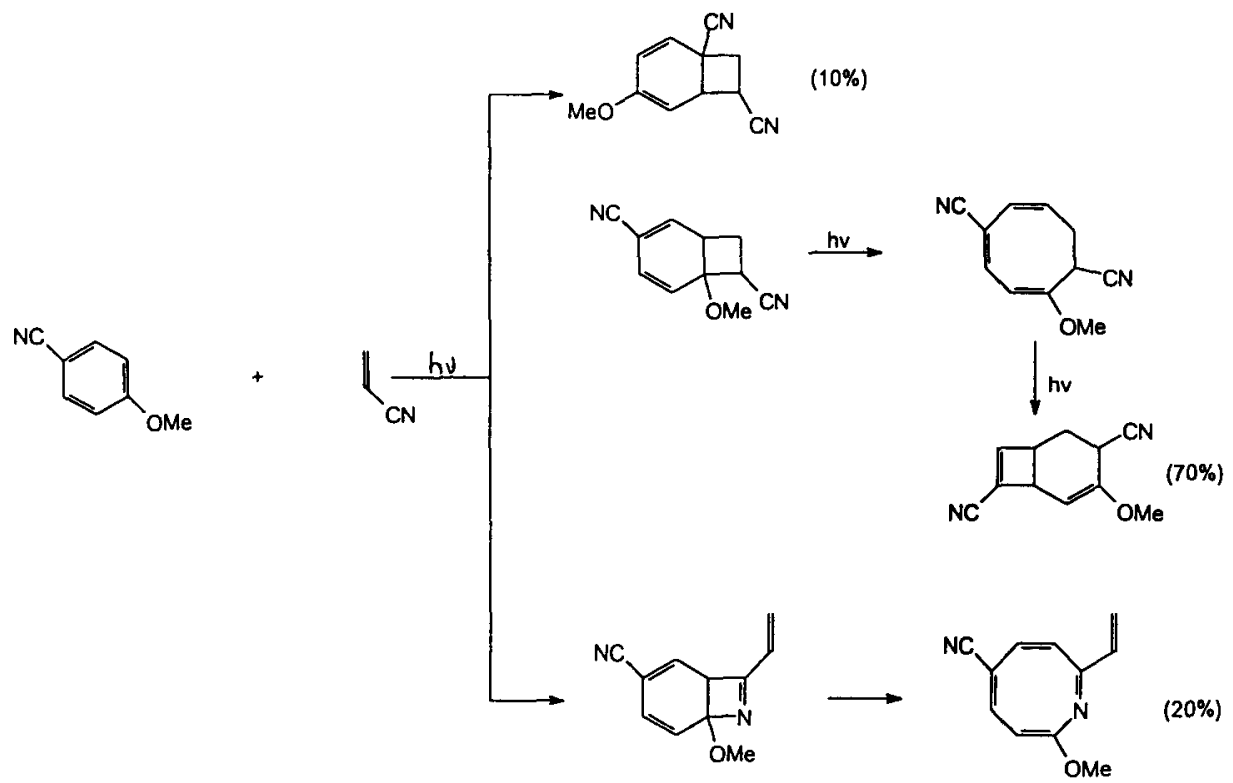

Scheme 3. Cycloaddition of acrylonitrile to 4-cyanoanisole.

the addends (Mattay 1985). Thus the ortho cycloaddition is favoured for addend pairs which have an electron donor-electron acceptor relationship whereas the meta process predominates or is the sole reaction for systems in which this feature is not evident. For example, enol ethers undergo cycloaddition at the 1,2-positions of benzonitrile, whereas cycloalkenes add at the 2,6-positions of toluene. When both electron donor and acceptor substituents (e.g. cyano and methoxy groups) are present on the benzene ring, then arene absorption allows sunlight excitation. Furthermore when the substituents are in conjugative positions (i.e. 1,2- or 1,4-substitution), the sites of ortho addition of electron donor and electron acceptor ethenes reflect the donor/acceptor natures of the arene substituents: this regiochemical feature is illustrated by the ortho cycloaddition products of acrylonitrile and ethyl vinyl ether with 2- and 4-cyanoanisoles shown in schemes 2 and 3 (Al-Jalal et al 1988). The formation of azacyclo-octatetraenes by the novel addition of the ethene cyano group to the benzene ring has been well-documented by Al-Jalal (1990). 
The primary cycloadduct from such 1,2-disubstituted benzenes is thermally labile since the common sigma bond in the bicyclic skeleton is weakened by the electron-donor and electron-acceptor groups. Disrotatory ring opening gives the cyclo-octatriene which absorbs in the $320 \mathrm{~nm}$ region and hence in sunlight undergoes disrotatory ring closure to yield the bicyclo[4.2.0] octa-2,7-diene as the sole product. This type of reaction also occurs intramolecularly with 4-phenoxybut-1-enes substituted with electron acceptor groups in the 2'- or 4'-positions (Cosstick et al 1987; Wagner and Nahm 1987). For example, 4-(2'-cyanophenoxy)but-1-ene undergoes an essentially

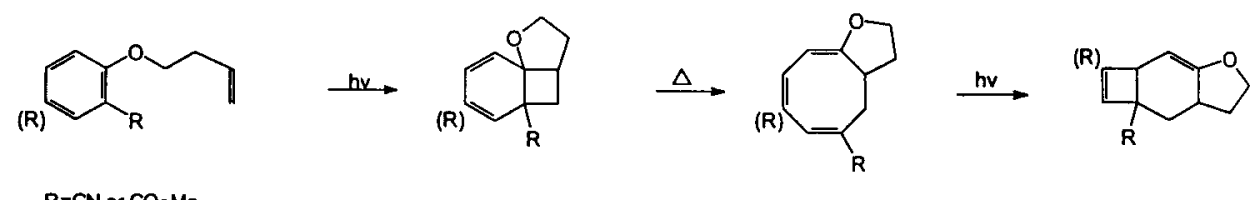

Scheme 4. Intramolecular cycloaddition of 4-phenoxybut-1-enes.

quantitative conversion to the 4-oxatricyclo[7.2.0.0.3,7 $]$ undeca-2,10-diene in sunlight (scheme 4). We have demonstrated that the formation of the triene can be quenched by common triplet quenchers but that the subsequent closure to the tricyclic system is not influenced by the presence of 1,3-dienes. The primary process is thus deduced to be a rare example of cycloaddition to the triplet state of the benzene ring, whereas the secondary photoreaction arises from the triene singlet state.

When both substituents of the benzene ring are electron donors or electron acceptors, excitation of the $\pi \pi^{*}$ transition of the arene is possible in sunlight. In such cases, if the ethene is a powerful electron acceptor or electron donor, respectively, substantially complete electron transfer can occur. For example, irradiation of methanol solutions of the 1,4-dimethoxybenzene-acrylonitrile system leads to substitution of the benzene ring by initial formation of the radical ion pair (Ohashi et al 1976, 1977). We have, however, adapted this system, as shown in scheme 5 , to yield the benzocyc-

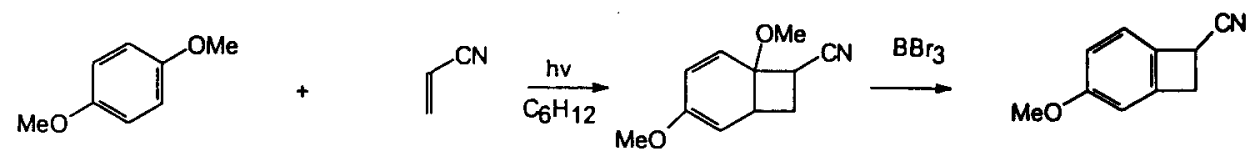

Scheme 5. Photocycloaddition of acrylonitrile to 1,4-dimethoxybenzene in non-polar solvents.

lobutene which has been used as a synthon in alkaloid and steroid synthesis (Kametani $1979,1981)$ by the simple expedient of mediating the electron transfer by using a nonpolar solvent. In this case, the reaction can be run to complete consumption of the arene, and decanting the reaction solution from the immiscible ethene photodimers (Al-Jalal and Gilbert 1983) allows an almost quantitative yield of the ortho cycloadduct to be obtained.

\subsection{1,3-(Meta) photocycloaddition}

The formation of meta photocycloadducts results in the greatest increase of "molecular complexity" (as defined by Bertz 1981) of any general reaction. The importance of 

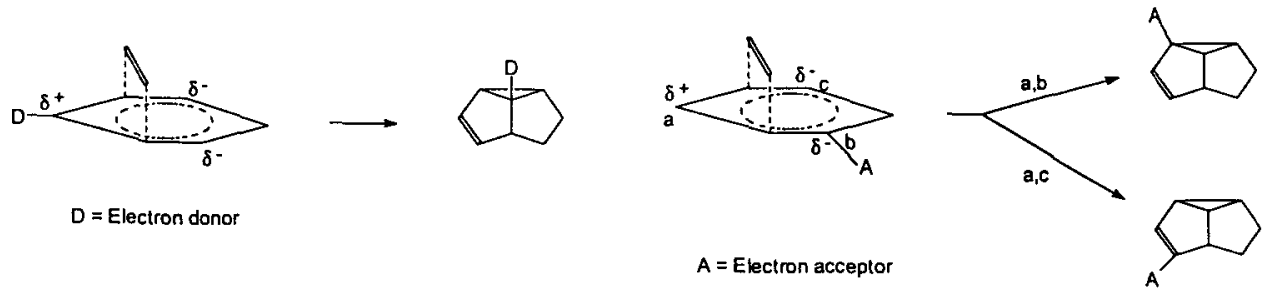

Scheme 6. Regiochemistry of meta cycloaddition of ethenes to substituted benzenes.

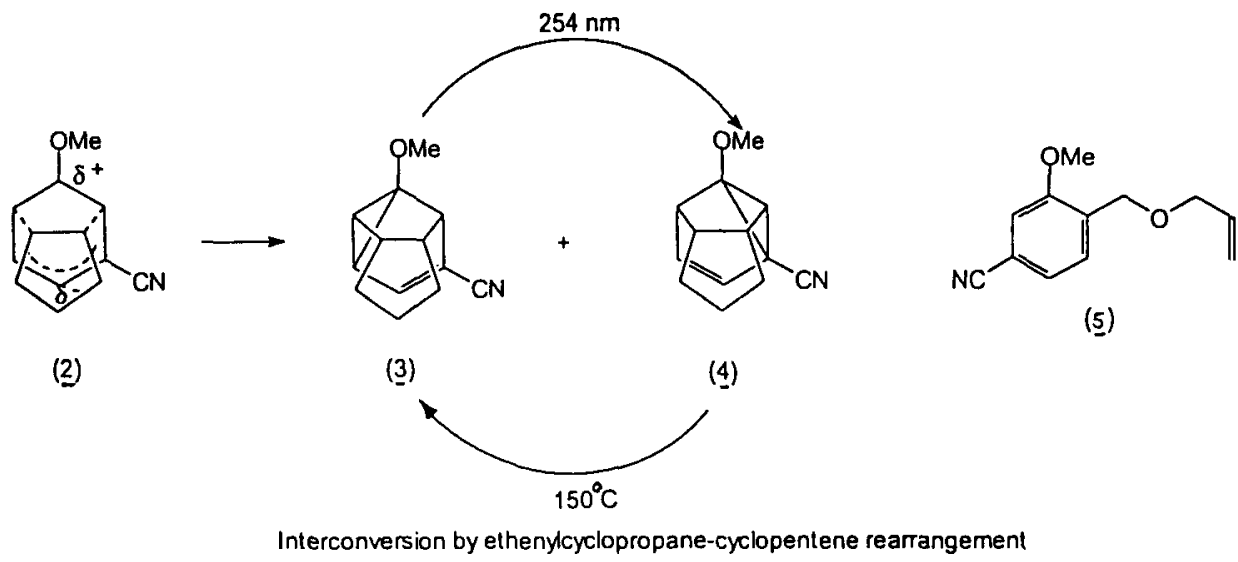

this process in synthetic organic chemistry has been elegantly demonstrated by Wender and co-workers by its use in a number of sequences to complex target molecules. As Wender notes "the value of the arene-alkene meta photocycloaddition (to synthetic organic chemistry) can be seen to arise from its capacity to produce a cycloadduct with three new rings and up to six new stereocentres, an impressive feat even when compared with the highly regarded Diels-Alder cycloaddition." (Wender 1990).

The basic feature of the widely accepted mechanism of meta photocycloaddition involves development of charge in the $S_{1}$ arene on approach of the $S_{0}$ ethene. The addends orient with respect to each other in order that the stabilisation of the developing charge centres in the $\mathrm{C}_{6}$ ring by the substituents is maximised: this accounts for the observed regiochemistry of the reaction (scheme 6). In 1,3-disubstituted benzenoid compounds such as 3-cyanoanisole, the charge stabilisation and hence the directing effects of the electron-withdrawing and electron-donating groups reinforce one another to give the orientation of the addends shown in 2. Delocalisation of the developing allylanion system by the cyano group promotes the intramolecular cyclisation giving the cyclopropane ring to favour formation of the 1-methoxy-4cyano- rather than the 1-methoxy-2-cyano-dihydrosemibullvalene. Exposure of the 3-cyanoanisole-cyclo-octene to sunlight yields the two isomers $\underline{3}$ and 4 in the respective ratio of 1:1.5 initially. This ratio changes with time reflecting the interconversion of the adducts photochemically and thermally by an ethenylcyclopropane-cyclopentene rearrangement. Indeed 3 or 4 may be obtained essentially free from the other isomer by the respective use of $254 \mathrm{~nm}$ radiation and heating at $150^{\circ} \mathrm{C}$. The formation of these meta photocycloadducts comprise the first reported example of sunlight-initiated 
meta photocycloaddition of an ethene to the benzene ring, but the process appears to be general for alkene additions to arenes 1,3-disubstituted with electron-donor and electron-acceptor groups and is currently being extended to the non-conjugated bichromophoric systems of type $\underline{5}$.

\section{Experimental}

All sunlight-induced reactions were carried out in a double-walled Pyrex apparatus. Solutions of the reactant(s) in cyclohexane or methanol under air were placed in the inner vessel and cooled water circulated through the outer jacket by convection. Product formation was monitored by GC and the photoadducts were isolated and identified as described previously for the reactions initiated by $254 \mathrm{~nm}$ radiation (see, for example, Al-Qaradawi et al 1992).

\section{Acknowledgements}

Finance from the University of Qatar (to SYA-Q) and a Postgraduate Studentship from the University of Reading (to DCB) is gratefully acknowledged.

\section{References}

Al-Jalal N 1990 J. Photochem. Photobiol. A54 99

Al-Jalal N and Gilbert A 1983 J. Chem. Res. 266

Al-Jalal N, Gilbert A and Heath P 1988 Tetrahedron 441449

Al-Qaradawi S Y, Cosstick K B and Gilbert A 1992 J. Chem. Soc., Perkin Trans. 11145

Andrews L J and Keefer R M 1964 Molecular complexes in organic chemistry (San Francisco: Holden-Day)

Angus H and Bryce-Smith D 1959 Proc. Chem. Soc. 326

Bertz S H 1981 J. Am. Chem. Soc. 1033599

Breuer A and Zincke Th 1878 Berichte 111403

Bryce-Smith D and Gilbert A 1977 Tetrahedron 332459

Bryce-Smith D, Gilbert A and Vickery B 1962 Chem. Ind. 2060

Buchi G, Inman C G and Lipinsky E S 1954 J. Am. Chem. Soc. 764327

Carassiti V 1988 EPA Newslett. (no. 33) 11

Cosstick K B, Drew M G B and Gilbert A 1987 J. Chem. Soc., Chem. Commun. 1867

Coyle J D (ed.) 1986 Photochemistry in organic synthesis: Special Publication No. 57 (London: The Royal Society of Chemistry)

Fritzsche J 1867 J. Prakt. Chem. 101333

Horspool W M (ed.) 1984 Synthetic organic photochemistry (New York: Plenum)

Hulsdunker A, Ritter A and Demuth M 1992 EPA Newslett. (no. 45) 23

Kametani T 1979 Pure Appl. Chem. 51747

Kametani T 1981 Tetrahedron 373

Klinger H and Slandke O 1891 Berichte 241340

Liebermann C 1877 Berichte 102177

Liebermann C 1890 Berichte 232510

Lomax N R and Narayanan V L 1984 Chemical structures of interest to the Division of Cancer Treatment (Bethesda, MD: National Cancer Institute)

Mattay J 1985 Tetrahedron 412393

Morsi S E and Abdel-Mottaleb M S A 1991 EPA Newslett. (no. 43) 11

Nasini R 1929 J. Chem. Soc. 996 
Ohashi M, Tanaka T and Yamada S 1976 J. Chem. Soc., Chem. Commun. 800

Ohashi M, Tanaka T and Yamada S 1977 Tetrahedron Lett. 3629

Paterno E and Chiefí G 1909 Gazz. Chim. Ital. 39341

Perkin W H 1881 J. Chem. Soc. 39409

Riiber C N 1902 Berichte 352908

Roth H D 1988 EPA Newslett. (no. 32) 1

Schenck G O and Steinmetz R 1960 Tetrahedron Lett. 831705

Sestini F 1866 Bull. Soc. Chim. 5202

Singer E 1986 EPA Newslett. (no. 26) 1

Turro N J 1965 Molecular photochemistry (New York: Benjamin)

Wagner P J and Nahm K 1987 J. Am. Chem. Soc. 109 4404, 6528

Wender P A 1990 Pure Appl. Chem. 621597 